\title{
SHORT TERM VARIABILITY OF LINE STRENGTHS \\ IN SOME OF AND WR STARS
}

\author{
W.G. Weller and S. Jeffers \\ Physics Department and CRESS \\ York University, Downsview, Ontario
}

\section{INTRODUCTION}

In recent years many observations of short time scale variability in a variety of spectral classes have been published. Some of these observations relate to phenomena which are reasonably well understood, as for example the Beta Canis Majoris stars, which are short period pulsators. In other cases, such as the Of and WR stars, the mechanism responsible for the variation is not fully understood. Models proposed to explain this variability include:- the binary hypothesis which ascribes the variations to fluctuations in gas streams in and around the members of a close binary system, and:- the pulsation hypothesis, which ascribes the variation in line strength to the presence of pulsational instability of massive carbon burning cores of evolved objects. The choice of the correct model is made difficult by the lack of an extended set of homogeneous observations. We must fulfil two conditions before making this choice. It is necessary to observe individual objects over extended periods of time at high dispersion to establish whether or not all WR's are binary systems. It is also required firmly to establish the temporal nature of the variations, since short term periodicities would tend to favor the core pulsation model.

Brucato, (1971), published a photographic survey of line strengths in nine of stars, and concluded that there was evidence for variability in several of his samples. Bhang, (1975), has published photoelectric line profiles for five WR stars. He similarly reported variations in the profiles of emission lines, but on longer time scales. Most of the remaining observations of rapid variability have been of isolated stars. We report here on an analysis of some data obtained for a sample of 10 of and 13 WR stars observed in May, June, and July 1974. A more complete analysis will be published elsewhere.

\section{OBSERVATIONS}

The spectra from which we obtain our results were taken with a SIT Vidicon described by Jeffers and Weller (1976). These spectra 
cover $150 . \mathrm{nm}$ at a dispersion of $\cdot 3 \mathrm{~nm}$ per channel and instrumental FWHM of 3 channels. The stellar lines were resolved, even for the coolest WR subclasses. Each data set comprised from 9 to 20 spectra, exposed for 30 seconds, with 2 minutes between exposures.

A 1ist of stars observed is as follows:-0f stars HD57060(=29CMa) HD124314, HD148937, HD150938, HD151804, HD153919(=3U1700-37), HD164794(=9Sgr), HD175754, HD188001(=9Sge); WN stars HD50896(=EZ CMa), HD86161, HD92740, HD151932; WC stars HD68273(=Gamma Ve1), HD92809, HD93131, HD136488, HD156385, HD164270, HD165273, HD168206(=CV Ser); and the WP starHD90657.

Spectral features were reduced in the following way. Two rectangular "filters" were defined, each centered on the feature to be measured. The widths of the filters were chosen to be 1.5 and $6.0 \mathrm{~nm}$. The ratio of the fluxes in these pass bands defines a line strength parameter which is independent of instrumental and atmospheric effects. Data were standardised with observations of stars of spectral types $\mathrm{B} 5$ and A0. These stars have absorption lines which are comparable in strength to the emission lines in the program stars, and can be assumed to have constant line profiles. Program stars were assumed not to vary if the ratio of fluxes did not vary any more than that for the standard stars (.014 mag. peak-to-peam). Figure 1 is a sample plot for two of stars indicated by this study to be constant in line strength. The upper line in each plot is a measure of the flux in a portion of the continuum. It varies strongly due to the effects of scintillation and guiding errors. This fluctuation is not seen in the ratio plot.

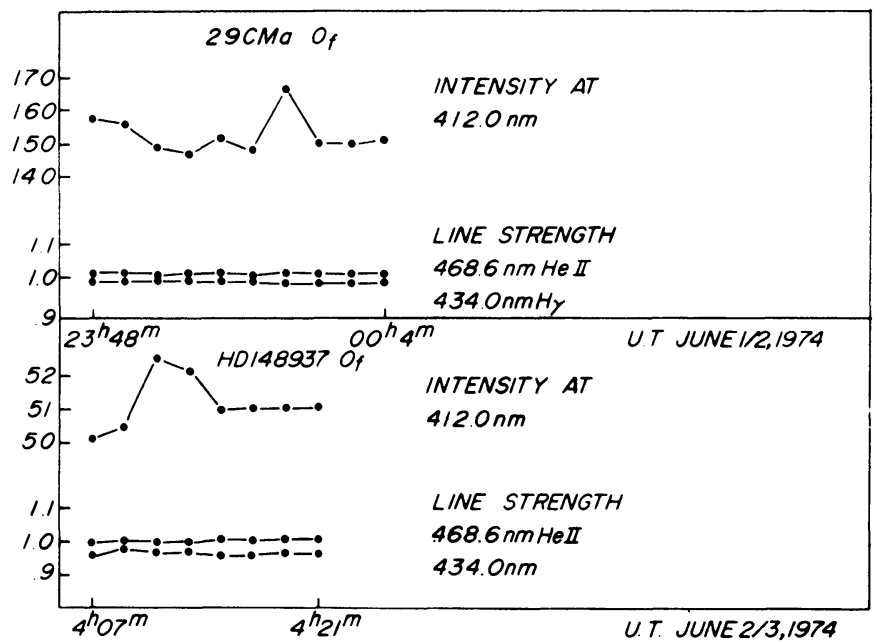

Fig. 1 Total intensity and flux ratios for HD148937 and $29 \mathrm{CMa}$

The nature of the plots is indistinguishable from that of the standard stars observed. Figure 2 presents data for HD50896 on 2 nights. On both nights both plotted lines show a gradual increase in strength over the 18 minutes of the observations. On the night of June $3 / 4$ there is evidence for irregular rapid fluctuations at $486.1 \mathrm{~nm}$ (HI, HeII). 


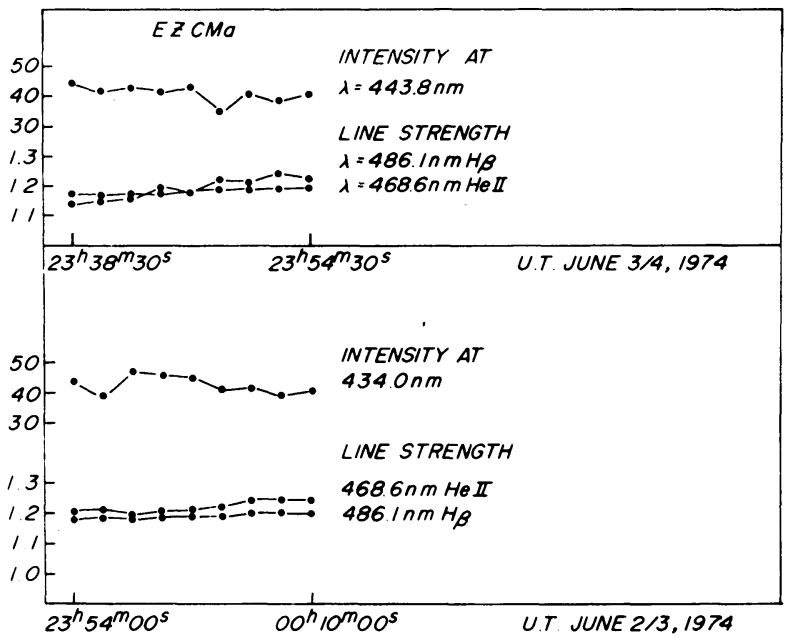

Fig. 2 Total intensity and flux ratios for HD50896 on 2 nights

\section{RESULTS AND DISCUSSION}

The results of the study are uniformly negative for all observed of stars, to the limit of our sensitivity. The WN stars in general also yield negative results, except for HD50896 a 1.01day binary (Kuhi, 1967). For the stars of type WC we find activity in several spectral features. HD68273 (Gamma Ve1), and HD92809 both vary at 468.6 $\mathrm{nm}$ (HeII), but apparently not at $434.0 \mathrm{~nm}$ (HI+HeII) HD156385 yields marginally positive results at $443.8 \mathrm{~nm}$ (CIV), but not at $465.0 \mathrm{~nm}$ (CIII). HD164270 appears to vary at $434 . \mathrm{nm}, 486.1 \mathrm{~nm}$ (HI+HeII) and 465.0 (CIII). No inference of periodic activity can be drawn on the basis of our data. There appears to be a trend in the following sense: The later the subclass, the more likely one is to find variability. The general lack of variability on time scales less than one hour in both $0 f$ and WN stars and the presence of such in the WC stars provides another observational distinction between the types.

\section{ACKNOWLEDGEMENTS}

The work described here was supported by the National Research Council of Canada. S.J. gratefully acknowledges travel support from the I.A.U. and the Physics Department of York University.

\section{REFERENCES}

Bhang, J.D.R., 1975, M.N.R.A.S., 170, 611.

Brucato, R., 1971, M.N.R.A.S., 153, 435.

Jeffers, S., and Weller, W., 1976, AEEP 40, 887.

Kuhi, L.V., 1967, PASP, 79, 57. 
DISCUSSION FOLLOWING WELLER AND JEFFERS

Thomas: I would agree with your second conclusion: Short-term variability of emission line strengths implies variability in energy input, which implies mechanical energy. But I disagree on the first, that no short-term variability implies radiative dominance. Note that all chromospheric theories based on a time-independent mechanical flux (i.e., generated by statistically-steady mechanical energy input) predict no variability, even though chromosphere-corona are dominated by mechanical energy input.

Underhill: Lack of observation of short-period variations in the intensity of a strong emission line is not evidence that short-period variations in the physical state of the atmosphere do not exist. For Of, WN stars $\mathrm{H} \alpha, \mathrm{H} \beta$ and He II 4686 may come chiefly from a large outer "nebula" which may not show irregular variations. However, an irregular input of energy and excitation into a low-lying, relatively dense chromosphere/corona may occur. To see this you must observe a line originating chiefly in that region. The visible spectral range does not offer suitable lines for the expected $\mathrm{Te}$, Ne ranges to be found just outside of and $W N$ photospheres. The choice of spectroscopic feature which is observed is very critical.

\section{Jeffers: Agreed.}

Noerdlinger: You cannot conclude from the presence of variations that there is mechanical energy input. Radiation pressure in lines can cause instabilities leading to mechanical waves. The instability was first discussed by E. A. Milne.

Jeffers: Agreed. Our statements regarding the interpretation of the data should be regarded as speculative. 